\title{
INTERPRETING THE BÖKSTEDT SMASH PRODUCT AS THE NORM
}

\author{
VIGLEIK ANGELTVEIT, ANDREW J. BLUMBERG, TEENA GERHARDT, \\ MICHAEL A. HILL, AND TYLER LAWSON \\ (Communicated by Michael A. Mandell)
}

Abstract. This paper compares two models of the equivariant homotopy type of the smash powers of a spectrum, namely the "Bökstedt smash product" and the Hill-Hopkins-Ravenel norm.

\section{INTRODUCTION}

In any symmetric monoidal category $\mathcal{C}$, a basic construction of a $C_{n}$-equivariant object arises from the smash power

$$
X \mapsto X^{\otimes n}=\underbrace{X \otimes X \otimes \ldots \otimes X}_{n} .
$$

The homotopical analysis of such constructions in the category of spaces and spectra is of classical importance. For instance, Steenrod operations arise from the cohomology of the homotopy coinvariants $X_{h \Sigma_{n}}^{\times n}$ of $X^{\times n}$ with respect to the $\Sigma_{n}$-action. More general power operations arise from the analysis of this kind of construction in the category of spectra.

Our focus in this paper is the analysis of the smash power construction in the equivariant stable category. An important application of the smash power construction of spectra arises in the study of trace methods for computing algebraic $K$-theory via topological cyclic homology and topological Hochschild homology. The target of the cyclotomic trace, $\mathrm{TC}(R)$, is constructed from an equivariant structure that arises on $\mathrm{THH}(R)$; $\mathrm{THH}(R)$ is built as a cyclic bar construction, with the $k$ th simplicial level the smash power $X^{\wedge k+1}$. Topological Hochschild homology is a cyclotomic spectrum and the construction of $\mathrm{TC}(R)$ relies on the cyclotomic structure on $\operatorname{THH}(R)$. The cyclotomic structure on $\operatorname{THH}(R)$ boils down to having good

Received by the editors November 12, 2015 and, in revised form, February 8, 2016.

2010 Mathematics Subject Classification. Primary 55P91.

The first author was supported in part by an NSF All-Institutes Postdoctoral Fellowship administered by the Mathematical Sciences Research Institute through its core grant DMS-0441170, NSF grant DMS-0805917, and an Australian Research Council Discovery Grant.

The second author was supported in part by NSF grant DMS-0906105.

The third author was supported in part by NSF DMS-1007083 and NSF DMS-1149408.

The fourth author was supported in part by NSF DMS-0906285, DARPA FA9550-07-1-0555, and the Sloan Foundation.

The fifth author was supported in part by NSF DMS-1206008 and the Sloan Foundation. 
models for the smash powers $R^{\wedge k}$ of a spectrum $R$ as a $C_{k^{-}}$-spectrum so that there are (suitably coherent) diagonal equivalences

$$
R \rightarrow \Phi^{C_{k}} R^{\wedge k} .
$$

Here $\Phi^{C_{k}}$ denotes the $C_{k}$-geometric fixed points.

Bökstedt introduced $\mathrm{THH}(R)$ before the invention of symmetric monoidal categories of spectra; he invented coherence machinery (which anticipated the development of symmetric spectra, as explained in [19]) to handle a point-set model of the smash product and proved that his model of smash powers had the right homotopy type. Bökstedt's construction produces the cyclotomic structure on $\mathrm{THH}(R)$. After the invention of modern categories of spectra, it became possible to give definitions of $\operatorname{THH}(R)$ that simply computed the cyclic bar construction in the usual way, circumventing the complexity of Bökstedt's coherence machinery. However, it was believed that the smash power in this context did not have the right fixed points (e.g., see [13, 2.5.9] and [7, §IX.3.9]).

Recently, the solution to the Kervaire invariant one problem by the fourth author, Hopkins, and Ravenel involved development of a "norm" functor $N_{H}^{G}$ from $H$ spectra to $G$-spectra which has the correct diagonal fixed points [10. In particular, there is an equivalence

$$
R \longrightarrow \Phi^{G} N_{e}^{G} R
$$

for any finite group $G$ and cofibrant $R$. When $G=C_{k}$, the underlying spectrum of $N_{e}^{C_{k}} R$ is precisely the smash power $R^{\wedge k}$.

This behavior strongly suggests that the norm should agree with Bökstedt's version of the smash powers. The purpose of this paper is to make this precise, by constructing an explicit comparison between the two as equivariant spectra. We build on earlier analysis by Shipley [19] which interpreted the Bökstedt construction in terms of a "detection functor" in symmetric spectra, and our approach is somewhat related to analysis done by Lunøe-Nielsen and Rognes [12.

To state our main results, we begin by fixing some notation and definitions for our indexing categories. Let $\mathcal{I}$ denote Bökstedt's indexing category, i.e., the category with objects $\mathbf{n}=\{1,2, \ldots, n\}$ and morphisms all injections. Let $\mathcal{J}$ denote the subcategory of $\mathcal{I}$ with the same objects but maps the subset inclusions. Notice that $\mathcal{J}$ has a unique map from $\mathbf{m}$ to $\mathbf{n}$ for $m<n$.

Recall the following definition of the Bökstedt smash product:

Definition 1.1. Let $X(1), X(2), \ldots, X(k)$ be symmetric spectra in spaces. We define the orthogonal Bökstedt smash product to be the orthogonal spectrum with $W$ th space

$$
\left(X(1) \stackrel{B}{\wedge} \ldots \wedge \wedge^{B} X(k)\right)_{W}=\underset{\left(\mathbf{n}_{1}, \ldots, \mathbf{n}_{\mathbf{k}}\right) \in \mathcal{I}^{\mathbf{k}}}{\operatorname{hocolim}} \Omega^{n_{1}+\ldots+n_{k}}\left(X(1)_{n_{1}} \wedge \ldots \wedge X(k)_{n_{k}} \wedge S^{W}\right) .
$$

Note that more generally we get a continuous functor from finite based $C W$ complexes to spaces by plugging in a $C W$-complex $A$ in place of $S^{W}$; by restriction, we can extract an orthogonal spectrum or symmetric spectrum.

Fix a complete $C_{k}$-universe $\mathcal{U}$. For our model of the equivariant stable category, we use the category of orthogonal $C_{k}$-spectra [14]. Specializing to the case of the smash power of a single spectrum $X$, observe that $X^{\wedge k}$ becomes a $C_{k}$-equivariant orthogonal spectrum indexed on $\mathcal{U}$ as we let $W$ vary through the finite-dimensional 
$C_{k}$-inner product spaces that embed in $\mathcal{U}$. Here $C_{k}$ acts by conjugation on the mapping space, permutation on the indexing category, and the action on $S^{W}$.

The following is the main theorem of this paper.

Theorem 1.2. Let $X$ be a cofibrant orthogonal spectrum, and let $\tilde{X}$ denote the underlying symmetric spectrum (of topological spaces). Then there is an isomorphism in the homotopy category of $C_{k}$-equivariant orthogonal spectra

$$
\tilde{X}^{\wedge k} \cong N_{e}^{C_{k}} X
$$

where $N_{e}^{C_{k}} X$ is the Hill-Hopkins-Ravenel norm.

Moreover, this isomorphism is induced by a zig-zag of maps natural in $X$.

To explain the proof, we introduce some further notation. Let $\mathbb{J}$ denote the category of finite-dimensional real inner product spaces $V$ in $\mathbb{R}^{\infty}$, with morphisms the inclusions $V \rightarrow W$ in $\mathbb{R}^{\infty}$. We regard this as a discrete (i.e., set-enriched) category. We will denote by $N_{e}^{C_{k}}$ both the norm from spectra to $C_{k}$-spectra as well as the norm from spaces to $C_{k}$-spaces; the usage will be clear from the context.

Let $\rho$ denote the regular representation of $C_{k}$. We now have the following main comparison diagram in the category of $C_{k}$-spaces:

$$
\begin{aligned}
& \left(\tilde{X}^{\wedge} \wedge k\right)_{W} \\
& \downarrow= \\
& \underset{\mathcal{I}^{k}}{\operatorname{hocolim}} \Omega^{n_{1}+\ldots+n_{k}}\left(\tilde{X}_{n_{1}} \wedge \ldots \wedge \tilde{X}_{n_{k}} \wedge S^{W}\right) \\
& \uparrow \Delta \\
& \underset{\mathcal{I}}{\operatorname{hocolim}} \Omega^{k n}\left(\tilde{X}_{n} \wedge \ldots \wedge \tilde{X}_{n} \wedge S^{W}\right)=\underset{\mathcal{I}}{\operatorname{hocolim}} \Omega^{k n}\left(N_{e}^{C_{k}}\left(\tilde{X}_{n}\right) \wedge S^{W}\right) \\
& \uparrow D_{1} \\
& \underset{\mathcal{J}}{\operatorname{hocolim}} \Omega^{k n}\left(N_{e}^{C_{k}}\left(\tilde{X}_{n}\right) \wedge S^{W}\right) \\
& \underset{\mathbb{J}}{\operatorname{\downarrow }} \underset{D_{2}}{\operatorname{Docolim}} \Omega^{V \otimes \rho}\left(N_{e}^{C_{k}}\left(X_{V}\right) \wedge S^{W}\right) .
\end{aligned}
$$

The map labeled $\Delta$ is the diagonal inclusion. The maps labeled $D_{i}$ are induced from the natural inclusion $\mathcal{J} \rightarrow \mathcal{I}$ and the functor $\mathcal{J} \rightarrow \mathbb{J}$ given (on objects) by $\mathbf{m} \mapsto \mathbb{R}^{m}$.

\section{A BRIEF REVIEW OF EQUIVARIANT ORTHOGONAL SPECTRA AND THE NORM}

We begin with a very rapid review of the details of the theory of equivariant orthogonal spectra that we need. The authoritative source on this subject is [14]; we also require a number of the refinements of the foundations developed in [10]. See also [1, §2] for a concise review.

2.1. Basic definitions. Fix a finite group $G$. A $G$-universe $\mathcal{U}$ is a $G$-inner product space isomorphic to $\bigoplus_{n \in \mathbb{N}} V$ for some finite-dimensional representation $V$ which contains a trivial representation [14, II.1.1]. We say $\mathcal{U}$ is complete when it contains 
all irreducible representations of $G$. Denote by $\mathcal{V}=\mathcal{V}(\mathcal{U})$ the collection of all finitedimensional $G$-inner product spaces that are isomorphic to sub- $G$-inner product spaces of $\mathcal{U}$ [14, II.2.1].

Given two representations $V, W \in \mathcal{V}$, we define $\mathscr{I}_{G}(V, W)$ to be the $G$-space of non-equivariant isometric isomorphisms $V \rightarrow W$. This construction allows us to regard $\mathcal{V}$ as the objects of a category $\mathscr{I}_{G}$ enriched in $G$-spaces. An orthogonal $G$-spectrum is now defined to be an enriched functor $X$ from $\mathscr{I}_{G}$ to the category of $G$-spaces equipped with an equivariant natural transformation

$$
X_{V} \wedge S^{W} \longrightarrow X_{V \oplus W}
$$

that satisfies the obvious transitivity property [14, §II.2].

Remark 2.1. A convenient aspect of this definition is that any continuous (i.e., enriched) functor from $G$-spaces of the homeomorphism type of finite $G$-CW complexes to $G$-spaces gives rise to an orthogonal $G$-spectrum by restriction of the domain; the required natural transformation comes from the continuity [2, A.3]. This observation is relevant to our work because many of the orthogonal $G$-spectra we study arise in this fashion.

The category of orthogonal $G$-spectra has a symmetric monoidal structure induced by the Day convolution. In order to describe this, we recall an alternate description of the category of orthogonal $G$-spectra [14, §II.4]. For representations $V$ and $W$, let $\mathscr{J}_{G}(V, W)$ denote the Thom space of the orthogonal complement $G$-bundle of linear isometries $V \rightarrow W$. We now have a category $\mathscr{J}_{G}$ with the same objects as $\mathscr{I}_{G}$, and an orthogonal $G$-spectrum is precisely an enriched functor from $\mathscr{J}_{G}$ to $G$-spaces. Now the Day convolution defines the symmetric monoidal structure, with unit the sphere spectrum $S$.

This description of orthogonal $G$-spectra is also useful because it provides an explicit description of the adjoint of the evaluation functors. For a $G$-representation $V$ and a based $G$-space $X$, we define the shift-desuspension spectrum $S^{-V} \wedge X$ to have $W$ th space $\mathscr{J}_{G}(V, W) \wedge X$ [14, II.4.6]. Of particular importance are the negative $V$-spheres $S^{-V}$.

We now turn to the homotopy theory of orthogonal $G$-spectra. The category of orthogonal $G$-spectra admits a model structure such that the homotopy category is equivalent to the equivariant stable category. To describe this model structure, we need to review the notion of equivariant stable homotopy groups.

The homotopy groups of an orthogonal $G$-spectrum $X$ are defined for a subgroup $H \subset G$ and an integer $q$ by

$$
\pi_{q}^{H}(X)= \begin{cases}\operatorname{colim}_{V \in \mathbb{J}} \pi_{q}\left(\left(\Omega^{V} X_{V}\right)^{H}\right) & \text { if } q \geq 0, \\ \operatorname{colim}_{\mathbb{R}^{-q} \subset V \in \mathbb{J}} \pi_{0}\left(\left(\Omega^{V-\mathbb{R}^{-q}} X_{V}\right)^{H}\right) & \text { if } q<0\end{cases}
$$

(see [14, §III.3.2]). We define the stable equivalences to be the maps $X \rightarrow Y$ that induce isomorphisms for all homotopy groups. There is a cofibrantly-generated monoidal model structure on orthogonal $G$-spectra in which the weak equivalences are the stable equivalences [14, §IV.2].

The stable equivalences are chosen so that the canonical map

$$
S^{-V} \wedge S^{V} \longrightarrow S^{0}
$$


is a stable equivalence for all $V$ [14, III.4.5]. In particular, this means that for any $G$-space $X$, we have a stable equivalence

$$
S^{-V} \wedge S^{V} \wedge X \longrightarrow \Sigma^{\infty} X
$$

We now recall an elementary properties of shifts of orthogonal spectra. For an orthogonal $G$-spectrum $X$, recall that $\Omega^{V} X$ denotes the orthogonal $G$-spectrum with

$$
\left(\Omega^{V} X\right)_{W}=\Omega^{V}\left(X_{W}\right) .
$$

Proposition 2.3. If $X$ is a $G$-space, then we have a natural stable equivalence

$$
S^{-V} \wedge X \longrightarrow \Omega^{V} \Sigma^{\infty} X
$$

of orthogonal $G$-spectra.

Proof. The map in question is the adjoint of the stable equivalence given by equation (2.2). Explicitly, the adjoint is the composite

$$
S^{-V} \wedge X \longrightarrow \Omega^{V} \Sigma^{V}\left(S^{-V} \wedge X\right) \longrightarrow \Omega^{V} \Sigma^{\infty} X
$$

where the first map is the unit of the $\left(\Sigma^{V}, \Omega^{V}\right)$ adjunction and the second map is $\Omega^{V}$ applied to the map in equation (2.2). The unit is a weak equivalence by [14, III.3.8] and $\Omega^{V}$ preserves weak equivalences by [14, III.3.9].

2.2. Canonical homotopy presentations. Up to stable equivalence, there is a convenient way of expressing the homotopy type of an orthogonal $G$-spectrum in terms of its spaces; this is referred to as the "canonical homotopy presentation" in [10, B.4.3]. Specifically, let

$$
V_{0} \subset V_{1} \subset \ldots \subset V_{n} \subset V_{n+1} \subset \ldots
$$

be an exhausting sequence of orthogonal $G$-representations (by which we mean that every finite-dimensional $G$-representation $V$ in $\mathcal{V}(\mathcal{U})$ can be embedded in some $V_{n}$ ) and write $W_{n}$ for the orthogonal complement of $V_{n}$ in $V_{n+1}$. We obtain zig-zags of the form

$$
S^{-V_{n}} \wedge X_{V_{n}} \longleftarrow S^{-\left(V_{n} \oplus W_{n}\right)} \wedge S^{W_{n}} \wedge X_{V_{n}} \longrightarrow S^{-V_{n+1}} \wedge X_{V_{n+1}}
$$

and taking the homotopy colimit over the first $m$ stages we get something equivalent to $S^{-V_{m}} \wedge X_{V_{m}}$. Therefore, we write

$$
\underset{V_{n}}{\operatorname{hocolim}} S^{-V_{n}} \wedge X_{V_{n}}
$$

to denote the homotopy colimit over this zig-zag. The result is stably equivalent to $X$; equation (2.4) is referred to as the "canonical homotopy presentation" of $X$.

Combining this with Proposition 2.3 gives a convenient description of orthogonal spectra; note that we are using the fact that homotopy colimits of orthogonal spectra can be computed levelwise using the Bousfield-Kan formula without any cofibrant replacement.

Lemma 2.5 (cf. [10, B.4.3]). If $X$ is an orthogonal $G$-spectrum and $\left\{V_{n}\right\}$ is an exhausting sequence of orthogonal $G$-representations, then we have a stable equivalence

$$
X \simeq\left(W \mapsto \underset{V_{n}}{\operatorname{hocolim}} \Omega^{V_{n}}\left(X_{V_{n}} \wedge S^{W}\right)\right)
$$


The exhausting sequences also provide a way to prove that the map $D_{2}$ of equation (1.3) is an equivalence. Any choice of exhausting sequence $V_{n}$ in $\mathbb{R}^{\infty}$ defines a functor $\mathcal{J} \rightarrow \mathbb{J}$ by

$$
\mathbf{n} \mapsto V_{n}
$$

Stated in this way, the sequence being exhausting is the same thing as the homotopy cofinality of this functor (e.g., see [11, A.5]).

Lemma 2.6. Let $X$ be an orthogonal $G$ spectrum. For any exhausting sequence $V_{n}$ in $\mathbb{R}^{\infty}$, the map

$$
\underset{n \in \mathcal{J}}{\operatorname{hocolim}} \Omega^{V_{n} \otimes \rho}\left(X_{V_{n} \otimes \rho} \wedge S^{W}\right) \stackrel{D_{2}}{\longrightarrow} \underset{V \in \mathbb{J}}{\operatorname{hocolim}} \Omega^{V \otimes \rho}\left(X_{V \otimes \rho} \wedge S^{W}\right)
$$

is an equivariant equivalence.

In particular, the map $D_{2}$ of equation (1.3) is always an equivalence.

Proof. The map $D_{2}$ is an equivariant equivalence because the inclusion $\mathcal{J} \rightarrow \mathbb{J}$ is homotopy cofinal and passage to fixed points commutes with filtered homotopy colimits.

2.3. The Hill-Hopkins-Ravenel norm. We now review the special case of the norm construction that we need. Given a non-equivariant orthogonal spectrum $X$, the smash power $X^{\wedge n}$ can be given a $C_{n}$-action and regarded as an orthogonal $C_{n}$-spectrum indexed on the universe containing only trivial representations (which we denote by $\mathbb{R}^{\infty}$ ). In order to obtain an orthogonal $C_{n}$-spectrum indexed on the complete $C_{n}$-universe $\mathcal{U}$, we simply apply the point-set change-of-universe functor $\mathcal{I}_{\mathbb{R}^{\infty}}^{U}[14, \S \mathrm{V} .1]$. This composite defines the norm as

$$
N_{e}^{C_{n}} X=\mathcal{I}_{\mathbb{R}^{\infty}}^{U} X^{\wedge n},
$$

and more generally the analogous construction can be used to define $N_{e}^{G} X$ for any finite group $G$. There is a more complicated general construction of the norm $N_{H}^{G} X$ which takes orthogonal $H$-spectra to orthogonal $G$-spectra for arbitrary subgroups $H \subset G$, but since we do not need that herein we refer the interested reader to 10 , $\S$ A.4] for details.

A key technical insight of Hill-Hopkins-Ravenel is that the norm $N_{e}^{G}$ preserves weak equivalences between cofibrant orthogonal $G$-spectra and, in fact, cofibrant (commutative) ring orthogonal $G$-spectra [10, $\S \mathrm{B} .2]$. That is, cofibrant replacement allows us to compute the derived functor of the norm.

We need one further fact about the norm, which relates the norm to a description in terms of the canonical homotopy presentation (equation (2.4)) and the norm functor on spaces [10, $\S 2.3 .2]$. For a space $A$, define

$$
N_{e}^{G}(A)=\bigwedge_{G} A
$$

regarded as a $G$-space via the permutation action. Then we have the following description of the norm:

$$
N_{e}^{G}(X)=\underset{V_{n}}{\operatorname{hocolim}} S^{-V_{n} \otimes \rho} \wedge N_{e}^{G}\left(X_{V_{n}}\right),
$$

where $\rho$ is the regular representation and $V_{n}$ is an increasing sequence of subspaces of $\mathbb{R}^{\infty}$. Note that as above, the hocolim notation hides the fact that the diagram has backwards weak equivalences. 
Since for any exhausting sequence $V_{n}$ for $\mathbb{R}^{\infty}$, the sequence $V_{n} \otimes \rho$ is exhausting for a complete universe, equation (2.7) and the argument for Lemma 2.5 provide another description of the norm.

Lemma 2.8. If $X$ is a cofibrant orthogonal spectrum, then there is a stable equivalence of orthogonal $G$-spectra

$$
N_{e}^{G}(X) \simeq\left(W \mapsto \underset{V \in \mathbb{J}}{\operatorname{hocolim}} \Omega^{V \otimes \rho}\left(N_{e}^{G}\left(X_{V}\right) \wedge S^{W}\right)\right) .
$$

\section{Changing Diagrams}

In this section, we show that the "change of indexing diagram" map $D_{1}$ is an equivariant stable equivalence.

To study $D_{1}$, we need to establish an equivariant version of Bökstedt's telescope lemma comparing homotopy colimits over $\mathcal{I}$ to homotopy colimits over $\mathcal{J}$ (i.e., telescopes). For this, we need to recall that in equivariant homotopy, there is a refined notion of "connectedness" which records the behavior of fixed points for subgroups (as opposed to the coarser notion of "inducing an isomorphism of homotopy groups through a range"). Let $\nu$ be a function from conjugacy classes of subgroups of $G$ to $\mathbb{N} \cup\{\infty\}$. A $G$-equivariant map $f: X \rightarrow Y$ is said to be " $\nu$-connected" if

$$
f^{H}: X^{H} \longrightarrow Y^{H}
$$

is $\nu(H)$-connected for all subgroups $H \subset G$. With this language, we can give an equivariant refinement of the telescope lemma. We use the formulation of the telescope lemma due to Schlichtkrull [18, 2.2]. In the following, we will refer to a functor $X: \mathcal{I} \rightarrow \operatorname{Top}_{G}$ as an $\mathcal{I}$ - $G$-space.

Lemma 3.1. Let $X$ be an $\mathcal{I}$-G-space and suppose that each morphism $\mathbf{n}_{\mathbf{1}} \rightarrow \mathbf{n}_{\mathbf{2}}$ in $\mathcal{I}$ with $n_{1} \geq n$ induces a $\nu_{n}$-connected map

$$
X\left(n_{1}\right) \longrightarrow X\left(n_{2}\right) .
$$

Then given any $m \geq n$, the natural map

$$
X(m) \longrightarrow \underset{\mathcal{I}}{\operatorname{hocolim}} X(i)
$$

is at least $\left(\nu_{n}-1\right)$-connected.

In particular, if for each $H, \nu_{n}(H)$ goes to infinity, then

$$
\underset{\mathcal{J}}{\operatorname{hocolim}} X(i) \longrightarrow \underset{\mathcal{I}}{\operatorname{hocolim}} X(i)
$$

is an equivariant equivalence.

Proof. Since the group $G$ does not act on $\mathcal{I}$, we know that the fixed points commute with the homotopy colimit: there is a natural equivalence

$$
\left.\underset{\mathcal{I}}{\operatorname{hocolim}}\left(X(i)^{H}\right) \stackrel{\simeq}{\longrightarrow} \underset{\mathcal{I}}{\operatorname{hocolim}} X(i)\right)^{H},
$$

where the map is induced from the natural inclusion $X(i)^{H} \rightarrow X(i)$.

By assumption, for all $\mathbf{n}_{\mathbf{1}} \rightarrow \mathbf{n}_{\mathbf{2}}$ and $n_{1} \geq n$ and for all $H$, we have

$$
X\left(n_{1}\right)^{H} \longrightarrow X\left(n_{2}\right)^{H}
$$


is $\nu_{n}(H)$-connected. By the usual telescope lemma [18, 2.2], we conclude that

$$
\left.X(m)^{H} \longrightarrow \underset{\mathcal{I}}{\operatorname{hocolim}} X(i)^{H} \stackrel{\simeq}{\longrightarrow} \underset{\mathcal{I}}{\operatorname{hocolim}} X(i)\right)^{H}
$$

is $\left(\nu_{n}(H)-1\right)$-connected.

The proof of the second part is immediate from the first, since by elementary cofinality arguments in $\mathcal{J}$, we see that under the given assumptions, the map

$$
\underset{\mathcal{J}}{\operatorname{hocolim}} X(j) \longrightarrow \underset{\mathcal{I}}{\operatorname{hocolim}} X(i)
$$

is infinitely equivariantly connected.

We will need some simple observations about the connectivity of a norm in spaces or of the norm of a map between spaces.

Lemma 3.2. The equivariant connectivity of $N_{e}^{G}(X)$ is given by $\nu_{X}$, where

$$
\nu_{X}(H)=\frac{|G|}{|H|}(\operatorname{conn}(X)+1)-1 \geq \frac{|G|}{|H|} \operatorname{conn}(X),
$$

while for a map $f: X \rightarrow Y$, the equivariant connectivity of $N_{e}^{G}(f)$ is given by $\nu_{f}$, where

$$
\nu_{f}(H)=\operatorname{conn}(f)+\left(\frac{|G|}{|H|}-1\right)(\operatorname{conn}(X)+1) .
$$

Proof. Both results rely on the diagonal: if $Y$ has the trivial action, the map

$$
Y^{|G| /|H|}=N_{H}^{G}(Y) \longrightarrow N_{e}^{G}(Y)
$$

is a homeomorphism on $H$-fixed points for any normal subgroup $H$. Standard arguments about the connectivity of non-equivariant smash powers of a map then show that the equivariant connectivity of $N_{e}^{G}(X)$ and $N_{e}^{G}(f)$ is given by the listed formulas. (See also [4, 3.12] for discussion of this phenomenon.)

Similarly, we recall a small result about the connectivity of an equivariant mapping space (e.g., see [9, 2.5]).

Lemma 3.3. Suppose $X$ and $Y$ are $G$-spaces with $X$ having the structure of an $H-C W$ complex. Then

$$
\operatorname{conn}\left(\operatorname{Map}(X, Y)^{H}\right) \geq \min _{K \subset H}\left(\operatorname{conn}\left(Y^{K}\right)-\operatorname{dim}\left(X^{K}\right)\right) .
$$

This will allow us to easily get very coarse lower bounds.

Proposition 3.5. Let $G=C_{k}$. For a space $X$, the suspension map

$$
E^{m \rho}: N_{e}^{G} X \longrightarrow \Omega^{m \rho} \Sigma^{m \rho} N_{e}^{G} X
$$

has connectivity at least

$$
\nu(H)=2 \frac{k}{|H|} \operatorname{conn}(X) .
$$

Proof. We use the equivariant Freudenthal suspension theorem 11 Recall that if $V$ is a representation of $G$, then the suspension map

$$
E^{V}: Y \longrightarrow \Omega^{V} \Sigma^{V} Y
$$

\footnotetext{
${ }^{1}$ For the interested reader, the first condition following is perhaps what one would expect: the connectivity is exactly what the usual Freudenthal suspension theorem would predict, just for each of the fixed points. The second condition is somewhat less obvious: this condition guarantees that the connectivity of $H$-equivariant maps between two spaces is the same as the ordinary maps between their $H$-fixed points.
} 
is $\nu_{V}$-connected for any $\nu_{V}$ satisfying

(1) For all $H$ such that $V^{H} \neq\{0\}$, we have $\nu_{V}(H) \leq 2 \operatorname{conn}\left(Y^{H}\right)+1$.

(2) For all $K \subset H$ with $V^{K} \neq V^{H}$, we have $\nu_{V}(H) \leq \operatorname{conn}\left(Y^{K}\right)$.

We now consider $V=m \rho$ and $Y=N_{e}^{G}(X)$. For all subgroups $H$ of $G, V^{H} \neq\{0\}$, and if $K \subsetneq H$, then $V^{K} \neq V^{H}$. If we let $\nu$ be as in the proposition statement, then the essential condition to check is the second one. So we must show that for all $K \subsetneq H$,

$$
\nu(H)=2 \frac{k}{|H|} \operatorname{conn}(X) \leq \operatorname{conn}\left(Y^{K}\right)=\frac{k}{|K|}(\operatorname{conn}(X)+1)-1 .
$$

However, since $K \neq H$, we know that this holds. Thus by the equivariant Freudenthal suspension theorem, the map $E^{m \rho}$ is $\nu$-connected.

To prove that the map $D_{1}$ is an equivariant equivalence, we will directly verify the needed connectivity hypotheses to apply Lemma 3.1

Theorem 3.6. Let $X$ be a cofibrant orthogonal spectrum with underlying symmetric spectrum $\tilde{X}$. Then the maps

$$
\underset{\mathcal{J}}{\operatorname{hocolim}} \Omega^{k n}\left(N_{e}^{C_{k}}\left(\tilde{X}_{n}\right) \wedge S^{W}\right) \stackrel{D_{1}}{\longrightarrow} \underset{\mathcal{I}}{\operatorname{hocolim}} \Omega^{k n}\left(N_{e}^{C_{k}}\left(\tilde{X}_{n}\right) \wedge S^{W}\right)
$$

are equivalences of $C_{k}$-spaces and therefore assemble to a stable equivalence of orthogonal $C_{k}$-spectra.

Proof. The first part of the conclusion implies the second; since we show that the map $D_{1}$ establishes level equivalences of equivariant orthogonal spectra, it gives an equivariant stable equivalence.

Since $\tilde{X}$ is the restriction of an orthogonal spectrum, it is a semistable symmetric spectrum [19]. Thus Shipley's version of Bökstedt's Telescope Lemma ([19, Corollary 3.1.7]) shows that $D_{1}$ induces an underlying equivalence.

Since both sides of the map preserve stable equivalences of orthogonal $G$-spectra (e.g., see [19, 2.1.9] for the right-hand side), we can simplify our analysis using the canonical homotopy presentation (equation (2.4)). Both the domain and range commute with filtered homotopy colimits in $X$ because $S^{n \rho}$ is compact, and the canonical homotopy presentation allows us to express $X$ as a filtered homotopy colimit of objects of the form $S^{-V} \wedge Y$. Therefore, it suffices to prove this result for orthogonal spectra of the form $X=S^{-V} \wedge Y$ with $Y$ cofibrant and $V$ a $d$ dimensional vector space. In this case, the underlying symmetric spectrum has $\tilde{X}_{n}=\mathscr{J}\left(V, \mathbb{R}^{n}\right) \wedge Y$.

To show that $D_{1}$ induces an equivariant equivalence, we verify that the $\mathcal{I}-C_{k^{-}}$ space

$$
\mathbf{n} \mapsto \Omega^{n \rho}\left(N_{e}^{C_{k}}\left(\tilde{X}_{n}\right) \wedge S^{W}\right)
$$

has the appropriate connectivity properties for Lemma 3.1. Here we have replaced the more traditional $\Omega^{k n}$ with the visibly equal functor $\Omega^{n \rho}$ to strengthen the equivariant connection in the reader's mind.

The presence of $S^{W}$ does not affect the structure of the argument; since it is constant relative to $\mathcal{I}$, having it will perform an affine shift of the connectivities we compute (in fact, making them increasingly connective). We can therefore get a lower bound by calculating in the case that $W=0$. 
The connectivity of $\tilde{X}_{n}$ is at least the connectivity $(2 n-2 d-1)$ of the Thom space $\mathscr{J}\left(V, \mathbb{R}^{n}\right)$. Similarly, the connectivity of the structure map $S^{m} \wedge \tilde{X}_{n} \rightarrow \tilde{X}_{m+n}$ is at least $(n+m-2 d)$.

Let $\mathbf{n}_{\mathbf{1}} \rightarrow \mathbf{n}_{\mathbf{2}}$ be a map in $\mathcal{I}$, and consider the following factorization of the structure map in the Bökstedt $\mathcal{I}$ - $G$-space:

$$
\begin{aligned}
& \Omega^{n_{1} \rho}\left(N_{e}^{C_{k}}\left(\tilde{X}_{n_{1}}\right) \wedge S^{W}\right) \\
& \Omega^{n_{1} \rho} \Omega^{\left(n_{2}-n_{1}\right) \rho} \sum^{\left(n_{2}-n_{1}\right) \rho}\left(N_{e}^{C_{k} \rho}\left(\tilde{X}_{n_{1}}\right) \wedge S^{W}\right) \underset{\Omega^{n_{2} \rho} N_{e}^{C_{k}} \sigma}{\longrightarrow} \Omega^{n_{2} \rho}\left(N_{e}^{C_{k}}\left(\tilde{X}_{n_{2}}\right) \wedge S^{W}\right)
\end{aligned}
$$

where $E^{\left(n_{2}-n_{1}\right) \rho}$ is the equivariant suspension and where $\sigma$ is the structure map in $X: \Sigma^{\left(n_{2}-n_{1}\right)} X_{n_{1}} \rightarrow X_{n_{2}}$.

The structure map in the $\mathcal{I}$ - $G$-space is at least as connected as

$$
\min \left(\operatorname{conn}\left(\Omega^{n_{1} \rho} E^{\left(n_{2}-n_{1}\right) \rho}\right), \operatorname{conn}\left(\Omega^{n_{2} \rho} N_{e}^{C_{k}} \sigma\right)\right),
$$

and we analyze each piece individually.

We first analyze the connectivity of $\Omega^{n_{1} \rho} E^{\left(n_{2}-n_{1}\right) \rho}$. Applying Proposition 3.5 and Lemma 3.3, we get a lower bound on the connectivity of the map $\Omega^{n_{1} \rho} E^{\left(n_{2}-n_{1}\right) \rho}$ :

$$
\begin{aligned}
& \nu_{1}(H)=\operatorname{conn}\left(\left(\Omega^{n_{1} \rho} E^{\left(n_{2}-n_{1}\right) \rho}\right)^{H}\right) \\
& \quad \geq \min _{K \subset H}\left(2 \frac{k}{|K|} \operatorname{conn}\left(\tilde{X}_{n_{1}}\right)-\frac{k}{|K|} n_{1}\right) \geq \frac{k}{|H|}\left(3 n_{1}-4 d-2\right) .
\end{aligned}
$$

Similarly, applying the norm $N_{e}^{C_{k}}$ to the structure map $\Sigma^{n_{2}-n_{1}} X_{n_{1}} \rightarrow X_{n_{2}}$ yields our map

$$
N_{e}^{C_{k}} \sigma: \Sigma^{\left(n_{2}-n_{1}\right) \rho} N_{e}^{C_{k}}\left(X_{n_{1}}\right) \cong N_{e}^{C_{k}}\left(\Sigma^{n_{2}-n_{1}} X_{n_{1}}\right) \longrightarrow N_{e}^{C_{k}}\left(X_{n_{2}}\right) .
$$

By Lemma 3.2, this norm produces a map that, on $H$-fixed points, has connectivity at least

$$
\begin{aligned}
\left(n_{1}+n_{2}-2 d\right)+\left(\frac{k}{|H|}-1\right)\left(\left(2 n_{1}-2 d-1\right)+\left(n_{2}-n_{1}\right)+1\right) & \\
& =\frac{k}{|H|}\left(n_{1}+n_{2}-2 d\right) .
\end{aligned}
$$

By Lemma 3.3 we can also estimate the connectivity of the $\left(n_{2} \rho\right)$-fold loops of this map, getting

$$
\begin{aligned}
\nu_{2}(H)=\operatorname{conn}\left(\left(\Omega^{n_{2} \rho} N_{e}^{C_{k}} \sigma\right)^{H}\right) & \\
& \geq \min _{K \subset H}\left(\frac{k}{|K|}\left(n_{1}+n_{2}-2 d\right)-\frac{k}{|K|} n_{2}\right)=\frac{k}{|H|}\left(n_{1}-2 d\right) .
\end{aligned}
$$

The factorization of the structure map above shows us that the structure map in the $\mathcal{I}$ - $G$-space has connectivity at least

$$
\nu(H)=\min \left(\nu_{1}(H), \nu_{2}(H)\right) \geq \min \left(3 n_{1}-4 d-2, n_{1}-2 d\right) \geq n_{1}-4 d-2
$$

(and usually a good bit more so).

Since this term goes to infinity, Lemma 3.1 implies that $D_{1}$ is an equivariant equivalence, as required. 


\section{Equivariance}

The maps $D_{1}$ and $D_{2}$ allow us to reduce from diagrams over $\mathcal{I}$ with a trivial action to diagrams over $\mathcal{J}$ with a trivial action of $C_{k}$. But one of the interesting aspects of the equivariant structure of the Bökstedt smash product is that $C_{k}$ acts on the diagram the homotopy colimit is indexed over. In this section, we show that the "diagonal" map $\Delta$ induces an equivariant equivalence. This allows us to reduce to the case of homotopy colimits on which the group acts trivially, as analyzed in the previous section.

The map $\Delta=\Delta_{1}$ is really part of a compatible family of maps, $\Delta_{i}$, indexed on the divisor poset of $k$. Analyzing the equivariant homotopy type of the Bökstedt construction is facilitated by a refinement of Hesselholt-Madsen's determination of the fixed points of the Bökstedt construction.

Suppose $k=d \cdot s$. Then we have a $C_{k}$-equivariant diagonal map

$$
\begin{aligned}
& \underset{\mathcal{I}^{d}}{\operatorname{hocolim}} \Omega^{s\left(n_{1}+\ldots+n_{d}\right)}\left(N_{C_{d}}^{C_{k}}\left(\bigwedge_{i=1}^{d} \tilde{X}_{n_{i}}\right) \wedge S^{W}\right) \\
& \downarrow \Delta_{d} \\
& \underset{\mathcal{I}^{k}}{\operatorname{hocolim}} \Omega^{n_{1}+\ldots+n_{k}}\left(\bigwedge_{i=1}^{k} \tilde{X}_{n_{i}} \wedge S^{W}\right) \text {. }
\end{aligned}
$$

The following proposition describes a key property of this generalized diagonal map. In this, we fix our model for the homotopy colimit as the two-sided bar construction.

Proposition 4.2. Suppose $k=d \cdot s$. Then the map of equation (4.1) induces a homeomorphism on passage to $C_{r}$-fixed points for $C_{s} \subseteq C_{r} \subseteq C_{k}$.

Proof. Our proof is a slight elaboration of that of Hesselholt-Madsen for the case $s=k[9, \S 2.4]$. The model for the homotopy colimit identifies the simplicial space realizing the homotopy colimit over $\mathcal{I}^{d}$ with a (nicely embedded) subsimplicial space of the homotopy colimit for $\mathcal{I}^{k}$, and as $s$ varies, these spaces are appropriately nested. Passage to fixed points commutes with geometric realization and with the products making up the two-sided bar construction for the homotopy colimit. This is what allows Hesselholt-Madsen to observe that the map $\Delta_{1}$ induces a homeomorphism on $C_{k}$-fixed points. Since $\Delta_{1}$ factors through $\Delta_{d}$ for any $d$, and since the inclusion is inducing a homeomorphism on fixed points in this case, we learn that $\Delta_{d}$ also induces a homeomorphism of $C_{k}$-fixed points (since these are really statements about fixed subspaces of a big ambient space). Downward induction on the group provides the rest of the result.

We use this proposition to show the following:

Theorem 4.3. The map

$$
\underset{\mathcal{I}}{\operatorname{hocolim}} \Omega^{k n}\left(N_{e}^{C_{k}} \tilde{X}_{n} \wedge S^{W}\right) \stackrel{\Delta_{1}}{\longrightarrow} \underset{\mathcal{I}^{k}}{\operatorname{hocolim}} \Omega^{n_{1}+\ldots+n_{k}}\left(\tilde{X}_{n_{1}} \wedge \ldots \wedge \tilde{X}_{n_{k}} \wedge S^{W}\right)
$$

induces a stable equivalence of equivariant orthogonal spectra.

Proof. Once again, we show that the map is a weak equivalence of $C_{k}$-spaces for each $W$; the maps therefore assemble to form a level equivalence and hence a stable equivalence of equivariant orthogonal spectra. 
We need to show that the map is an equivalence on $C_{s}$-fixed points for each $s \mid k$. Consider the composite

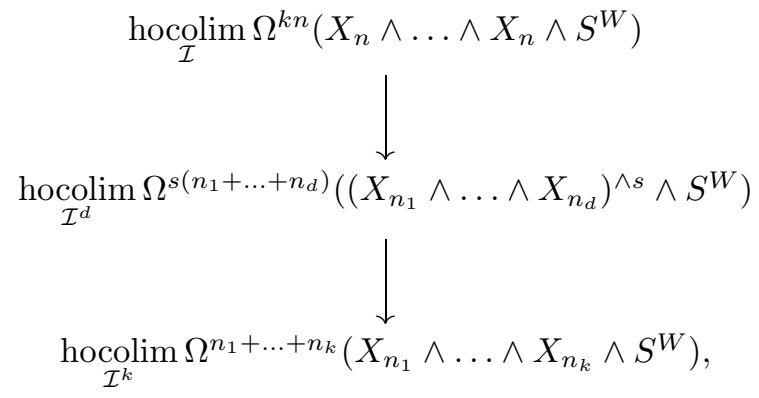

where $k=d \cdot s$ as before. The second map is an equivalence on $C_{s}$-fixed points by Proposition 4.2. and we claim that the first map is an equivalence on $C_{s}$-fixed points as well. Consider the diagram

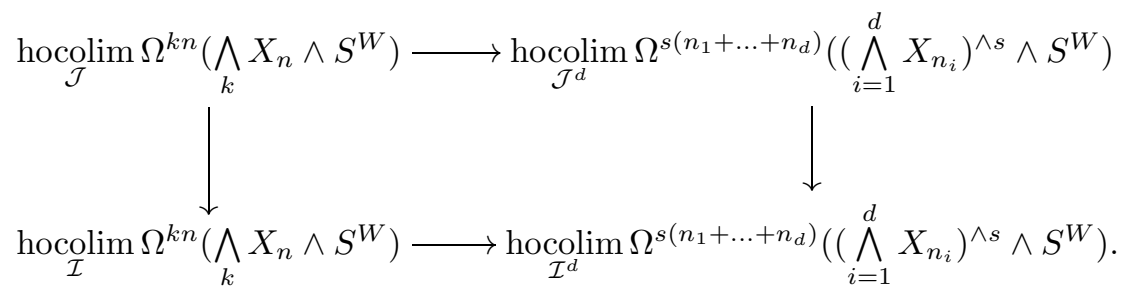

The point is that $C_{s}$ acts trivially on all the indexing categories in the diagram, and the diagonal map $\mathcal{J} \rightarrow \mathcal{J}^{d}$ is homotopy cofinal. Since the passage to fixed points commutes with these homotopy colimits, we conclude that the top horizontal map is a $C_{s}$-equivalence.

The left-hand side vertical map is an equivariant equivalence by Section 3, the right-hand side vertical map is an equivariant equivalence for the same reason, using Fubini's theorem for homotopy colimits (e.g., see [6, 24.9]).

This completes the proof of Theorem 1.2. We have shown that the vertical maps in the main comparison diagram (equation (1.3) ) from Section 1 do indeed assemble to weak equivalences of equivariant orthogonal spectra. We have also identified the orthogonal $C_{k}$-spectrum at the bottom of the comparison diagram with the spectrum $N_{e}^{C_{k}}(X)$.

\section{APPENDIX A. On EQUIVARIANT HOMOTOPY COLIMITS AND COFINALITY}

Our proof that the map $\Delta_{1}$ is an equivalence was a direct argument. A more categorical approach uses an equivariant notion of homotopy cofinality first described by Villarroel-Flores [20. For our purposes, we write $G$-category to denote an internal category in $G$-sets. If $\mathcal{C}$ is a $G$-category, then a $G$-equivariant $\mathcal{C}$-shaped diagram is a functor

$$
F: \mathcal{C} \longrightarrow \text { Top }
$$


together with compatible natural transformations for each $g \in G$,

$$
g: F \Rightarrow F \circ g,
$$

where the identity element in $G$ acts as the identity and where in $F \circ g$, we use the $G$-action on $\mathcal{C}$ [20. In particular, for each $c \in \mathcal{C}, F(c)$ is a $S t a b(c)$-space and $F(g \cdot c)$ is the homeomorphic $g S t a b(c) g^{-1}$-space given by pulling back along the conjugation isomorphism.

The action of $G$ on the diagram in any equivariant $\mathcal{C}$-shaped diagram induces an action of $G$ on the ordinary homotopy colimit (see Section 4 of 20]), and for the prototypical example of $G=C_{k}, \mathcal{C}=\mathcal{I}^{k}$, and the functor being Bökstedt's construction, this is the usual action of $C_{k}$ on THH.

The fixed points of a $G$-category $\mathcal{C}$ is an ordinary category, and the restriction of a $G$-functor $F$ to $\mathcal{C}^{G}$ is an ordinary functor into $G$-spaces. This lets us easily compare the fixed points of the homotopy colimit over $\mathcal{C}$ to the homotopy colimit of the fixed points.

Lemma A.1. If $\mathcal{C}$ is a $G$-category and $F: \mathcal{C} \rightarrow$ Top is an equivariant $G$-shaped diagram, then the natural maps of $G$-spaces

$$
\underset{\mathcal{C}^{G}}{\operatorname{hocolim}} F(c)^{G} \longrightarrow \underset{\mathcal{C}^{G}}{\operatorname{hocolim}} F(c) \longrightarrow \underset{\mathcal{C}}{\operatorname{hocolim}} F(c)
$$

are equivalences on $G$-fixed points.

Proof. We choose as a model for hocolim the two-sided bar construction $B(*, \mathcal{C}, F)$. This is the geometric realization of a simplicial space, and since the $G$-fixed point functor commutes with geometric realization, we need only show that this equality is true for the $r$-simplices of the bar construction for each $r$. Since we are in spaces, the fixed point space of a product is the product of the fixed point spaces, and we conclude

$$
\underset{\mathcal{C}}{(\operatorname{hocolim}} F(c))^{G} \cong \underset{\mathcal{C}^{G}}{\operatorname{hocolim}} F(c)^{G} .
$$

In fact, since we have chosen a particular model, these spaces are actually homeomorphic. The right most term is visibly $\left(\operatorname{hocolim}_{\mathcal{C}^{G}} F(c)\right)^{G}$.

The following trivial generalization of the preceding result is surprisingly useful:

Corollary A.2. Suppose that $\mathcal{C}$ is a $G$-category and $\mathcal{D}$ is a $G$-subcategory such that $\mathcal{C}^{G} \subset \mathcal{D}$. Then for any equivariant $\mathcal{C}$-shaped diagram $F$ from $\mathcal{C}$ to $T$ op, we have an equivalence of fixed points

$$
\left.(\underset{\mathcal{D}}{\operatorname{hocolim}} F(d))^{G} \longrightarrow \underset{\mathcal{C}}{\operatorname{hocolim}} F(c)\right)^{G}
$$

Proof. The assumptions on $\mathcal{C}$ and $\mathcal{D}$ ensure that $\mathcal{C}^{G}=\mathcal{D}^{G}$. The result follows from identifying both with this common colimit.

To complete the proof of the equivalence of $\Delta_{1}$, we can directly appeal to an equivariant form of Quillen's Theorem A. 
Theorem A.3 ([20, Theorem 6.3]). Let $F: \mathcal{C} \rightarrow \mathcal{D}$ be a functor such that for every $d \in \mathcal{D}$, the over-category $F \downarrow d$ is Stab $(d)$-equivariantly contractible. Then $F$ induces an equivalence on homotopy colimits.

In our context, this version of Quillen's Theorem A shows that the natural map $\mathcal{J} \rightarrow \mathcal{J}^{k}$ is equivariantly cofinal. By Lemma 3.1 and the commutativity of the obvious diagram of homotopy colimits, we conclude that $\Delta_{1}$ is an equivariant equivalence.

\section{ACKNOWLEDGEMENTS}

The authors would like to thank the American Institute of Mathematics (AIM) for its support through the SQuaREs program and MSRI for its hospitality while some of this work was being done. The authors would like to thank Mike Mandell for his assistance, as well as Lars Hesselholt and Mike Hopkins for interesting and useful conversations. The paper was improved by useful corrections provided by an anonymous referee.

\section{REFERENCES}

[1] Andrew J. Blumberg and Michael A. Mandell, The homotopy theory of cyclotomic spectra, Geom. Topol. 19 (2015), no. 6, 3105-3147, DOI 10.2140/gt.2015.19.3105. MR3447100

[2] Andrew J. Blumberg, Continuous functors as a model for the equivariant stable homotopy category, Algebr. Geom. Topol. 6 (2006), 2257-2295, DOI 10.2140/agt.2006.6.2257. MR2286026

[3] M. Bökstedt, Topological Hochschild homology, Preprint.

[4] M. Bökstedt, W. C. Hsiang, and I. Madsen, The cyclotomic trace and algebraic K-theory of spaces, Invent. Math. 111 (1993), no. 3, 465-539, DOI 10.1007/BF01231296. MR.1202133

[5] Bjørn Ian Dundas, Relative K-theory and topological cyclic homology, Acta Math. 179 (1997), no. 2, 223-242, DOI 10.1007/BF02392744. MR1607556

[6] Wojciech Chachólski and Jérôme Scherer, Homotopy theory of diagrams, Mem. Amer. Math. Soc. 155 (2002), no. 736, x+90, DOI 10.1090/memo/0736. MR 1879153

[7] A. D. Elmendorf, I. Kriz, M. A. Mandell, and J. P. May, Rings, modules, and algebras in stable homotopy theory, Mathematical Surveys and Monographs, vol. 47, American Mathematical Society, Providence, RI, 1997. With an appendix by M. Cole. MR1417719

[8] Thomas G. Goodwillie, Relative algebraic K-theory and cyclic homology, Ann. of Math. (2) 124 (1986), no. 2, 347-402, DOI 10.2307/1971283. MR855300

[9] Lars Hesselholt and Ib Madsen, On the K-theory of finite algebras over Witt vectors of perfect fields, Topology 36 (1997), no. 1, 29-101, DOI 10.1016/0040-9383(96)00003-1. MR.1410465

[10] M. A. Hill, M. J. Hopkins, and D. C. Ravenel, On the non-existence of elements of Kervaire invariant one, Preprint, arXiv:0908.3724.

[11] John A. Lind, Diagram spaces, diagram spectra and spectra of units, Algebr. Geom. Topol. 13 (2013), no. 4, 1857-1935, DOI 10.2140/agt.2013.13.1857. MR3073903

[12] Sverre Lunøe-Nielsen and John Rognes, The topological Singer construction, Doc. Math. 17 (2012), 861-909. MR3007679

[13] Ib Madsen, Algebraic K-theory and traces, Current developments in mathematics, 1995 (Cambridge, MA), Int. Press, Cambridge, MA, 1994, pp. 191-321. MR1474979

[14] M. A. Mandell and J. P. May, Equivariant orthogonal spectra and S-modules, Mem. Amer. Math. Soc. 159 (2002), no. 755, x+108, DOI 10.1090/memo/0755. MR.1922205

[15] M. A. Mandell, J. P. May, S. Schwede, and B. Shipley, Model categories of diagram spectra, Proc. London Math. Soc. (3) 82 (2001), no. 2, 441-512, DOI 10.1112/S0024611501012692. MR.1806878

[16] M. A. Mandell and B. Shipley, A telescope comparison lemma for THH, Topology Appl. 117 (2002), no. 2, 161-174, DOI 10.1016/S0166-8641(00)00121-8. MR1875908

[17] Randy McCarthy, Relative algebraic K-theory and topological cyclic homology, Acta Math. 179 (1997), no. 2, 197-222, DOI 10.1007/BF02392743. MR.1607555 
[18] Christian Schlichtkrull, Units of ring spectra and their traces in algebraic K-theory, Geom. Topol. 8 (2004), 645-673 (electronic), DOI 10.2140/gt.2004.8.645. MR2057776

[19] Brooke Shipley, Symmetric spectra and topological Hochschild homology, K-Theory 19 (2000), no. 2, 155-183, DOI 10.1023/A:1007892801533. MR1740756

[20] R. Villarroel-Flores, The action by natural transformations of a group on a diagram of spaces, Preprint, arxiv.org 0411502.

Department of Mathematics, Australian National University, Canberra, ACT 0200, Australia

E-mail address: vigleik.angeltveit@anu.edu.au

Department of Mathematics, University of Texas, Austin, Texas 78712

E-mail address: blumberg@math.utexas.edu

Department of Mathematics, Michigan State University, East Lansing, Michigan 48824

E-mail address: teena@math.msu.edu

Department of Mathematics, University of California los Angeles, Los Angeles, CALIFORNIA 90095

E-mail address: mikehill@math.ucla.edu

Department of Mathematics, University of Minnesota, Minneapolis, Minnesota 55455

E-mail address: tlawson@math.umn.edu 